\title{
SÍNDROME DE KOUNIS, UN INFARTO OLVIDADO: REPORTE DE CASO
}

\author{
KOUNIS SYNDROME, A FORGOTTEN INFARCTION: CASE REPORT
}

1 Residente de Segundo año, Medicina Interna

Universidad de la Habana

${ }^{2}$ Médico y Cirujana. Universidad Industrial de

Santander

Colombia,

Bucaramanga. Santander.

Colombia

Santander.

3 Médico General Universidad

Autónoma de

Bucaramanga. Bucaramanga.

Santander. Colombia

Dr. Edgar Leonardo Fontecha1, Dr. Nathalia Jácome Pérez², Dr. Oscar Leonardo Torra³.

\section{RESUMEN}

La anafilaxia es una causa poco frecuente de síndrome coronario agudo por vasoespasmo con o sin la presencia de enfermedad coronaria subyacente. Presentamos el caso de un síndrome de Kounis en un paciente masculino sin factores de riesgo conocidos para enfermedad coronaria quien presentó un síndrome coronario agudo con elevación de ST que requirió manejo con adrenalina, soporte vital básico e ingreso a Unidad de Cuidados Intensivos; con arteriografía coronaria sin evidencia de enfermedad subyacente.

\section{ABSTRACT}

Correspondencia a: Dr. Oscar Leonardo Torra Correo electrónico: otorra@ unab.edu.co

Telefono: +573162490509 ORCID: 0000-0002-7131-7457

Palabras clave: Anafilaxia, Síndrome de Kounis, Clindamicina, Arteriografía

Keywords: Anaphylaxis, Kounis syndrome, Clindamycin, Arteriography

Procedencia y arbitraje: no comisionado, sometido a arbitraje externo.

Recibido para publicación: 8 de mayo 2021

Aceptado para publicación: 21 de mayo de 2021

Citar como:

Leonardo Fontecha E.L., Jácome Pérez N.,Leonardo Torra O. SÍNDROME DE KOUNIS, UN INFARTO OLVIDADO: reporte de caso: Rev Cient Cienc Med. 2021; 24(1): 196-200

Anaphylaxis is a rare cause of vasospasm acute coronary syndrome with or without the presence of underlying coronary disease. We present the case of Kounis syndrome in a male patient with no known risk factors for coronary heart disease who presented with acute coronary syndrome with elevation of ST that required management with epinephrine, basic life support, and admission to the Intensive Care Unit; with coronary arteriography without evidence of underlying disease.

\section{INTRODUCCIÓN}

a relación entre alergia e infarto se ha observado durante mucho tiempo, pero no fue hasta 1991 que los médicos griegos Kounis y Zavras propusieron el concepto de "angina alérgica" causada por la constricción de las arterias coronarias inducida por histamina'. Posteriormente fueron vinculados signos y síntomas clínicos (cardiacos y alérgicos), complementados con pruebas de laboratorio, alteraciones electrocardiográficas, cambios contráctiles en el ecocardiograma y ausencia de lesiones obstructivas en la arteriografía coronaria. El síndrome de Kounis o angina alérgica, por tanto, se basa en la presentación de una reacción alérgica (de hipersensibilidad, anafiláctica o anafilactoide) que desencadena un síndrome coronario agudo ${ }^{2}$.

Se estima que la prevalencia de reacciones alérgicas en general es alrededor del 1,6\% a lo largo de la vida. La incidencia de anafilaxia con síntomas cardiovasculares asociados es de 8 por 100.000 habitantes en Estados Unidos y Europa ${ }^{3}$. La mayor parte de la información sobre este síndrome se basa únicamente en reportes de casos individuales. Probablemente muchos pacientes siguen sin ser diagnosticados ${ }^{4,5}$.

El mecanismo fisiopatológico de este síndrome se explica por el desarrollo de una reacción alérgica después del contacto con un alimento específico, inhalante, factor ambiental, después de una picadura o mordedura de insectos o después de la administración de la droga (shock anafiláctico), lo que da como resultado la acción de las células y mediadores alérgicos (como histamina, factor activador de plaquetas, proteasas, citocinas, quimiocinas), generando la contracción de las arterias coronarias o el comienzo de desintegración y activación de la placa aterosclerótica ${ }^{2}$.

Inicialmente, se distinguieron 2 tipos en función de la coexistencia de una enfermedad arterial coronaria ${ }^{1,2}$. El tipo 1 se diagnostica en personas que no presentaron lesiones en las arterias coronarias o factores de riesgo cardiovascular, por lo que se produce un 
espasmo de las arterias coronarias con o sin elevación de los marcadores bioquímicos de necrosis miocárdica. El tipo 2 ocurre en personas con aterosclerosis de las arterias coronarias donde la placa aterosclerótica se inestabiliza y rompe en el curso de una reacción alérgica. Con el tiempo, también se distinguió el tipo 3 en pacientes portadores de stents recubiertos con fármacos ${ }^{4}$. El diagnóstico precoz de la enfermedad permite un tratamiento adecuado.

El objetivo de este manuscrito es reportar un caso de Síndrome de Kounis con el fin de evidenciar la importancia en la sospecha diagnóstica, así como el reconocimiento oportuno y precoz del cuadro clínico que permita definir el tratamiento apropiado. Presentamos un caso secundario a clindamicina donde se realizó el manejo apropiado para limitar la reacción inmune que lo desencadena, evitando así complicaciones que puedan poner en riesgo la vida del paciente.

\section{PRESENTACIÓN DEL CASO}

Paciente masculino en la octava década de la vida con antecedentes de importancia de hipertensión arterial crónica (HTA), enfermedad renal crónica IIIA (ERC), hipotiroidismo, trombosis venosa profunda (TVP) crónica en miembros inferiores sin anticoagulación; sin antecedentes alérgicos conocidos quien consultó a consulta externa de cirugía vascular por presentar un cuadro clínico de 2 meses de evolución caracterizados por dolor, prurito, ardor, eritema, edema y calor en tercio distal de miembro inferior derecho. Posterior a la valoración fue remitido al servicio de urgencias con diagnóstico de erisipela, por lo que se inició tratamiento antibiótico con clindamicina y ampicilina sulbactam. Posterior a la administración de la primera dosis de clindamicina, el paciente presentó síntomas disautonómicos como ser hipotensión y síncope por lo que se consideró probable respuesta anafiláctica a clindamicina y se administraron 300 mg intravenosos de hidrocortisona más $0,5 \mathrm{mg}$ subcutáneos de adrenalina. Durante la monitorización con cardiovisoscopio se evidenció en DII un supradesnivel del segmento ST; con confirmación electrocardiográfica de síndrome coronario agudo con elevación de ST (SCACEST) en cara inferior y cara lateral (IAM TIPO II); (Ver Figura 1). Se considera que se trata de Síndrome de Kounis y se indica manejo antiisquémico pleno, priorizando el manejo para el choque anafiláctico y reanimación hídrica agresiva. A los 15 minutos del evento se evidencia normalización electrocardiográfica, aplanamiento del segmento ST en cara inferior con inversión de onda T en cara antero-septal (Ver Figura 2); Se obtuvieron biomarcadores con delta positivo. Llevado a arteriografía coronaria prioritaria (primeras 12 horas del evento) no se evidencian lesiones angiográficamente significativas (Ver Figura 3). Por lo que el paciente es trasladado a $\mathrm{UCl}$ por alto riesgo de arritmia letal o desenlace fatal.

Al ingreso a la unidad dependiente de soporte vasopresor, sin ángor torácico; química sanguínea dentro de límites normales. Se comenta con el servicio de infectología, quien indica manejo con linezolid. Durante la estancia se identifica en el cardiovisoscopio frecuentes episodios de extrasístoles ventriculares bigeminadas y trigeminadas, con posteriores episodios de bradicardia sinusal hasta 46 LPM. Se procede a comentar con el servicio de cardiología en donde indica toma de ecocardiograma transtorácico, electrocardiograma holter. Reporte de ecocardiograma transtorácico: función ventricular izquierda normal (FEVI 57 \%), sin trastornos de la motilidad, Al levemente dilatada e IA trivial. Reporte de electrocardiograma holter (24 horas): ritmo sinusal predominante durante el registro, frecuencia cardiaca promedio 90 LPM, mínima 45 LPM y máxima 135 LPM, no pausas significativas, bloqueo AV de primer grado pr 226 mseg. Bloqueo de rama QRS 177 mseg, no se evidenciaron arritmias auriculares. Frecuentes extrasístoles ventriculares monomórficas con periodo de acoplamiento mayor a uno en bigeminismo y trigeminismo. Variabilidad de la frecuencia cardiaca, intervalo QTC (466 mseg) y segmento ST normales. Por evolución clínica favorable, fue suspendida la doble antiagregación plaquetaria y el paciente fue trasladado a sala general a las 48 horas, se completó esquema antibiótico con Linezolid intrahospitalario satisfactoriamente durante 10 días posteriores a los cuales egresó de la institución en buenas condiciones generales. 


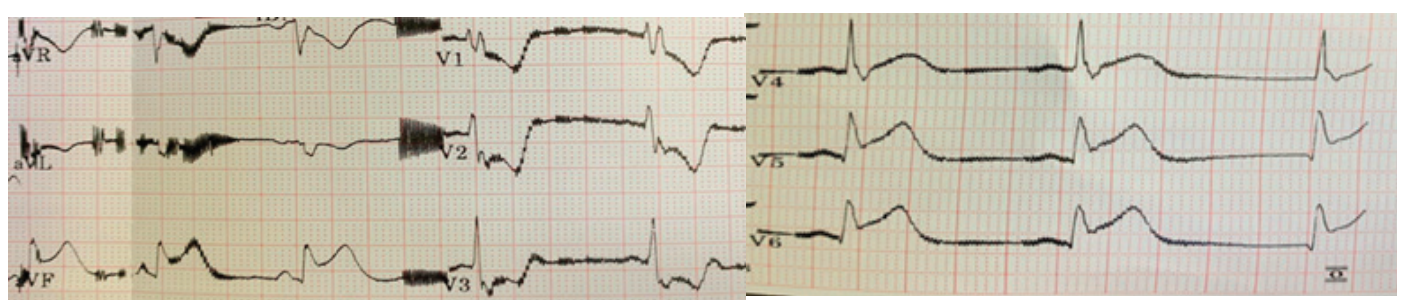

Figura 1: Electrocardiograma que evidencia IAMCEST en cara inferior y cara lateral

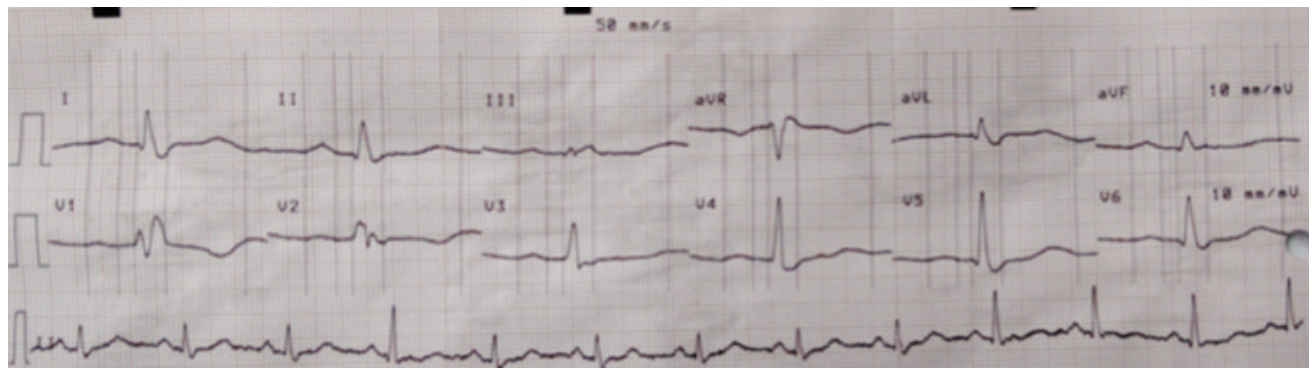

Figura 2: Electrocardiograma donde se evidencia aplanamiento del segmento ST en cara inferior con inversión de onda $T$ en cara antero-septal

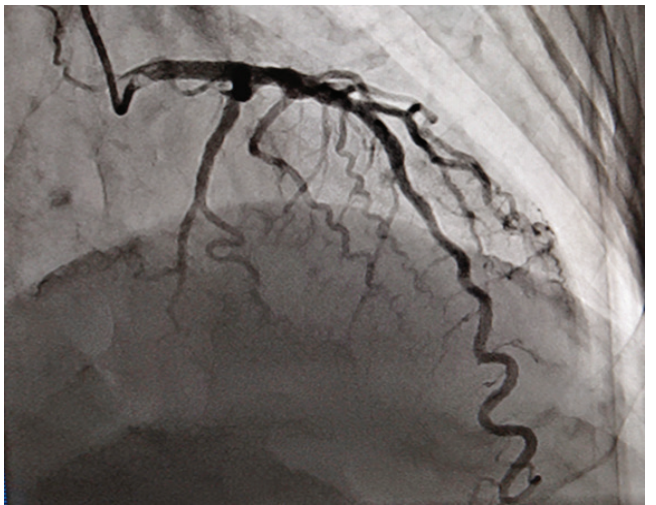

Figura 3: Arteriografía coronaria sin evidencia de lesiones angiográficamente significativas

\section{DISCUSIÓN}

El síndrome de Kounis es una entidad patológica que solo se conoce durante 28 años ${ }^{2}$. La información sobre sus síntomas, diagnóstico y tratamiento proviene principalmente de informes de casos o pequeños grupos de pacientes ${ }^{5,6}$. El diagnóstico adecuado de esta enfermedad y el tratamiento de la reacción alérgica, así como un síndrome coronario puede ser una tarea difícil para los médicos ${ }^{7}$. Los signos y síntomas vienen dados por urticaria, erupción cutánea, dificultad para respirar, hipoxemia, debilidad, dolor de cabeza, náuseas, vómitos, piel pálida, sudoración, taquicardia o bradicardia, edema, angioedema, shock y paro cardíaco repentino, estos son típicos de las reacciones alérgicas ${ }^{2,8}$. Síntomas asociados con un síndrome coronario y el síndrome de Kounis están dados por; dolor anginoso palpitaciones, disnea, lipotimia, arritmias y muerte súbita ${ }^{8}$. Algunos de ellos, como disnea y síntomas disautonómicos estuvieron presentes en el paciente. La manifestación electrocardiográfica del síndrome suele ser cambios dinámicos en el ST, aunque se han informado episodios de arritmias supraventriculares y ventriculares ${ }^{2}$. El diagnóstico del síndrome requiere una evaluación cardíaca y alergológica urgente. En el abordaje del laboratorio del síndrome de Kounis es importante una elevación en la concentración de marcadores bioquímicos de necrosis miocárdica. Se utiliza un perfil de lípidos para evaluar el riesgo cardiovascular. También se recomienda el diagnóstico alergológico que incluye $\lg \mathrm{E}$ total $\mathrm{y}$ específica ${ }^{2}$. La cuantificación de histamina es una ayuda desde el punto de vista teórico. Sin embargo, hay que tener en cuenta que tiene una vida media muy corta lo que limita su detección sérica ${ }^{3}$. Al contrario, la triptasa es una biomolécula más estable, con más especificidad para la activación de mastocitos, siendo una herramienta útil para diferenciar eventos anafilácticos de los fenómenos anafilactoides ${ }^{4,6}$. El ecocardiograma y la arteriografía coronaria son necesarias para la identificación de anormalidades de la contractilidad y para valorar la anatomía 
coronaria y diferenciar entre el tipo I y tipo II, y guiar la terapia. En nuestro caso se llevó de forma emergente sin evidencia de lesiones angiográficamente significativas.

Las pautas para el manejo son muy amplias porque se carece de evidencia para desarrollar guías ${ }^{7}$. Entonces, se recurre a extrapolar las recomendaciones de expertos para cada una de las entidades por aparte: infarto y reacciones alérgicas.

Inicialmente debemos evaluar rápidamente vía aérea, el estado respiratorio y el estado hemodinámico. Si hay compromiso de la vía aérea, se debe garantizar una adecuada oxigenación, se usará mascarilla facial, intubación endotraqueal si es preciso.

Las reacciones alérgicas graves causan hipotensión arterial, situación por la cual esta contraindica el uso de medicamentos vasodilatadores o antihipertensivos. Ante la hipotensión en el contexto de anafilaxia se debe hacer reanimación hídrica conservadora con controles estrictos del balance hídrico, por el riesgo de edema pulmonar asociado a disfunción ventricular en el infarto ${ }^{8}$.

El uso de nitratos, en pacientes con síndrome de Kounis, no hipotensos, puede ser adecuado siempre y cuando la tensión arterial del paciente lo permita ${ }^{3,4}$. Como medida complementaria el uso de antagonistas de los canales de calcio debe tenerse en cuenta como opción terapéutica, ya que tienen un papel importante en el tratamiento del infarto causado por vasoespasmo ${ }^{5}$.

El ácido acetilsalicílico como antiagregante plaquetario se recomienda en todo paciente con alta probabilidad de síndrome coronario en ausencia de contra indicaciones. No obstante, es necesario evaluar riesgobeneficio del medicamento, ya que está asociado con hipersensibilidad, por lo cual siempre que se considere la posibilidad de que este medicamento pueda empeorar el proceso alérgico, se recomienda desensibilizar o usar terapia alternativa como clopidogrel ${ }^{7}$.

La adrenalina es el tratamiento recomendado para la anafilaxia ${ }^{9}$, a pesar de que en presencia de síndrome coronario está asociado a isquemia, arritmias y vasoespasmo coronario. En cuanto al uso de betabloqueadores este fármaco puede disminuir los efectos benéficos de la adrenalina, ya que se opone al sistema simpático, contrario a los beneficios demostrados en el manejo del síndrome coronario agudo ${ }^{9,10}$.

El pronóstico a largo plazo es muy bueno con una bajo índice de falla cardiaca, solamente durante la fase aguda puede desarrollar arritmias, trombos, edema de pulmón, sin embargo la muerte es muy rara. Aunque no se han publicado recurrencias, la exposición repetida al alérgeno podría causar de nuevo el cuadro $^{10}$.

\section{CONCLUSIÓN}

El síndrome de Kounis no es una entidad infrecuente, pero sí sub-diagnosticada. Es necesario sospecharla en pacientes que presentan un síndrome coronario agudo, acompañado de signos y síntomas producidos por una reacción anafiláctica, para definir de forma oportuna el manejo y tratamiento adecuados que permitan disminuir el riesgo de complicaciones potencialmente mortales. En la actualidad no existen guías específicas para el tratamiento de este síndrome, motivo por el cual su abordaje depende de la forma de presentación, comorbilidades y disponibilidad de un grupo médico interdisciplinario, que incluya monitoreo y vigilancia en una unidad de cuidado intensivo. 


\section{REFERENCIAS}

1. Arora S, Patel R, Fadila M, Wool K. The atopic heart: a curious case of coronary hypersensitivity. Neth J Med. 2016; 74(3): 1302.

2. Reber LL, Hernandez JD, Galli SJ. The pathophysiology of anaphylaxis. J Allergy Clin Immunol. 2017; 140(2): 335-48.

3. Ramírez-Morejón A, Rodríguez-Moya V, Consuegra-Espinosa O, Puerto-Pérez T, GiroPuig D, et all. A propósito del síndrome de Kounis. Arch méd Camagüey. 2020; 24(2): 27792.

4. Kounis NG. Kounis syndrome: an update on epidemiology, pathogenesis, diagnosis and therapeutic management. Clin Chem Lab Med. 2016; 54(10):1545-59.

5. Renda F, Landoni G, Trotta F, Piras D, Finco G, et al. Kounis Syndrome: An analysis of spontaneous reports from international pharmacovigilance database. Int J Cardiol. 2016; 203: 217-20.
6. Kounis NG, Zavras GM. Allergic angina and allergic myocardial infarction. Circulation. 2016; 94(7): 1789.

7. Villamil Munévar PA, Sánchez-Solanilla LF. Síndrome de Kounis o angina alérgica. Rev colomb cardiol. 2017; 24(4):382-7.

8. Kounis NG, Kouni SN, Koutsojannis CM. Myocardial infarction after aspirin treatment, and Kounis syndrome. J R Soc Med. 2015;98(6):296.

9. Omri M, Kraiem H, Mejri O, Naija M, Chebili N. Management of Kounis syndrome: two case reports. J Med Case Rep. 2017; 11(1): 145.

10. Peláez-Pérez JM, Sánchez Casado M, Álvarez-Twose I, Kounis NG. Síndrome de Kounis del tipo II inducido por amoxicilina-ácido clavulánico complicado con encefalopatía hipóxico-isquémica durante anestesia general. Rev Esp Anestesiol Reanim. 2021; 68(3): 161-4. 